\title{
Genome-wide copy number variations as molecular diagnostic tool for cutaneous intermediate melanocytic lesions: a systematic review and individual patient data meta-analysis
}

\author{
Chiel F. Ebbelaar ${ }^{1,2}$ (D) Anne M. L. Jansen ${ }^{1} \cdot$ Lourens T. Bloem $^{2} \cdot$ Willeke A. M. Blokx $^{1}$ \\ Received: 17 December 2020 / Revised: 8 March 2021 / Accepted: 30 March 2021 / Published online: 13 April 2021 \\ (C) The Author(s) 2021
}

\begin{abstract}
Cutaneous intermediate melanocytic neoplasms with ambiguous histopathological features are diagnostically challenging. Ancillary cytogenetic techniques to detect genome-wide copy number variations (CNVs) might provide a valuable tool to allow accurate classification as benign (nevus) or malignant (melanoma). However, the CNV cut-off value to distinguish intermediate lesions from melanoma is not well defined. We performed a systematic review and individual patient data meta-analysis to evaluate the use of CNVs to classify intermediate melanocytic lesions. A total of 31 studies and 431 individual lesions were included. The CNV number in intermediate lesions (median 1, interquartile range [IQR] $0-2)$ was significantly higher $(p<0.001)$ compared to that in benign lesions (median 0 , IQR $0-1)$ and lower $(p<0.001)$ compared to that in malignant lesions (median 6 , IQR 4-11). The CNV number displayed excellent ability to differentiate between intermediate and malignant lesions $(0.90,95 \%$ CI $0.86-0.94, p<0.001$ ). Two CNV cut-off points demonstrated a sensitivity and specificity higher than $80 \%$. A cut-off of $\geq 3$ CNVs corresponded to $85 \%$ sensitivity and $84 \%$ specificity, and a cut-off of $\geq 4$ CNVs corresponded to $81 \%$ sensitivity and $91 \%$ specificity, respectively. This individual patient data meta-analysis provides a comprehensive overview of CNVs in cutaneous intermediate melanocytic lesions, based on the largest pooled cohort of ambiguous melanocytic neoplasms to date. Our metaanalysis suggests that a cut-off of $\geq 3 \mathrm{CNVs}$ might represent the optimal trade-off between sensitivity and specificity in clinical practice to differentiate intermediate lesions from melanoma.
\end{abstract}

Keywords Melanocytic $\cdot$ Melanocytoma $\cdot$ Ambiguous $\cdot$ Intermediate $\cdot$ Copy number variation $\cdot$ Meta-analysis

\section{Introduction}

Cutaneous melanocytic neoplasms include various tumor types with clinical behavior ranging from indolent to invasive [1]. Histopathologic evaluation is usually sufficient for classification as either benign (nevus) or malignant (melanoma). However, a minority displays ambiguous histopathological features, not allowing definite classification. Studies of preneoplastic melanocytic lesions have shown that intermediate stages exist

Willeke A. M. Blokx

W.A.M.Blokx@umcutrecht.nl

1 Department of Pathology, Division of Laboratories, Pharmacy and Biomedical Genetics, University Medical Center Utrecht, P.O. Box 85500, 3508 Utrecht, GA, Netherlands

2 Division of Pharmacoepidemiology and Clinical Pharmacology, Utrecht Institute for Pharmaceutical Sciences, Utrecht University, Utrecht, Netherlands in the progression from nevus to melanoma, associated with the acquisition of pathogenic genomic aberrations [2, 3]. These observations challenge the notion that melanocytic neoplasms can only be benign or malignant. Therefore, one of the significant changes in the recently updated World Health Organization (WHO) classification of skin tumors is the classification of melanocytic tumors in nine pathways with four-step progression models [4]. As such, the group of intermediate tumors has expanded, for which the term "melanocytoma" has been proposed with two different grades. These lesions present a diagnostic challenge even for expert dermatopathologists $[5,6]$. Importantly, incorrect classification might result in either preventable disease progression or substantial unnecessary costs, psychological stress, and additional surgery. Therefore, various ancillary cytogenetic techniques are employed to help distinguish nevi from melanomas, based on the fact that melanomas usually harbor copy number variations (CNVs) whereas nevi do not (or show specific isolated abnormalities) [7]. Cytogenetic techniques such as comparative genomic hybridization (CGH) 
array and single-nucleotide polymorphism (SNP) array can detect $\mathrm{CNVs}$ genome-wide, resulting in improved diagnostic accuracy in ambiguous melanocytic lesions compared to FISH [8, 9]. Thus, CNVs might provide a valuable tool to allow accurate classification. However, to what extent intermediate lesions carry CNVs has not been well established yet, and a CNV cut-off value to distinguish them from melanoma is not well defined. Therefore, we performed a systematic review and individual patient data meta-analysis to evaluate the use of CNVs to classify intermediate melanocytic lesions.

\section{Method}

\section{Search and study selection}

Embase and PubMed were systematically searched for primary research articles published in English until September 2020, using the terms "ambiguous," "atypical," "borderline," "dysplastic," "intermediate," "spitzoid," "uncertain," or "unclassified," paired with major keywords for melanocytic lesions (including melanocytic "lesion," "tumor," "proliferation," "neoplasm," "nevus," "nevi," "melanoma," "melanocytoma," "MELTUMP," "spitz*," "STUMP”). These results were then overlapped with the MeSH/Emtree terms for DNA copy number variations: "copy number"; "CNA"; "CNV"; "chromosomal aberration, duplication, amplification, deletion, alteration"; "comparative genomic hybridization"; "CGH"; or "SNP array." After duplicate removal, unique records were screened for eligibility based on title and abstract first and full-text records thereafter by two authors (CE, WB) using Rayyan for systematic reviews [10]. Differences were discussed until consensus was reached or through input from a third author (AJ). Last, backward and forward snowballing of included articles was employed to identify additional articles of interest.

\section{Eligibility criteria and outcomes of interest}

Articles were included when reporting on intermediate cutaneous melanocytic lesions using molecular techniques to identify genome-wide CNVs, such as CGH array and SNP array. Studies using next-generation sequencing (NGS) were included when using panels or computational methods allowing genome-wide copy number calling [11, 12]. Studies using FISH or multiplex ligation-dependent probe amplification (MLPA) were excluded since these techniques do not screen genome-wide for CNVs. Case reports, abstracts, poster presentations, and articles reporting on non-cutaneous melanomas or melanoma cell lines were excluded. The primary outcomes of interest were the number of CNVs and the type of chromosomal aberrations. Secondary outcomes were clinical follow-up, genomic aberrations, and histopathological characteristics.

\section{Data collection and CNV count}

CNVs were identified on individual lesion level. Authors were contacted to obtain individual patient data or additional information if needed. Two authors (CE, AJ) independently performed a CNV count based on the reported chromosomal aberrations using a predefined ruleset. Segmental gains, losses, high-level amplifications, aneuploidy, and polyploidy were each counted as one CNV. Homozygous loss was counted as two CNVs. CNVs considered insignificant in some studies because of their association with generally benign behavior, such as loss of 3p21 (BAP1 gene) and gain of 11p (HRAS gene), were included in the CNV count for uniformity. Chromosomal fusions for which both fusion partners were known were counted as one CNV since they result from one translocation event. Copy-neutral loss of heterozygosity (CN$\mathrm{LOH}$ ) was registered separately since it is not accompanied by actual copy number changes. In contrast, chromothripsis can comprise many $\mathrm{CNVs}$ but constitutes one tumor event. Therefore, chromothripsis was also registered separately. CNV counts were crosschecked against the reported number of CNVs when available. Ambiguities were resolved via contacting corresponding authors, discussion until consensus, or input from a third author (WB).

\section{Recategorization and reclassification of lesions}

All lesions were reviewed in-depth by two authors (CE, WB) and were recategorized and reclassified according to the 2018 WHO classification of skin tumors. Ambiguous lesions were recategorized as either "benign," "intermediate," or "malignant" hierarchically based on (1) provided clinical followup, (2) WHO definition, and (3) histopathology and additional case information. Ambiguous or benign lesions with metastatic disease beyond regional lymph nodes during follow-up were recategorized as malignant. Positive sentinel lymph node biopsies were not considered sufficient proof of malignancy since a minority of benign lesions occasionally display such behavior [13]. Per WHO definition, BAPl-inactivated nevi (BIN), deep penetrating nevi (DPN), cellular blue nevi $(\mathrm{CBN})$, and congenital nevi with proliferative nodules (CNPN) were recategorized as low-grade intermediate. BAP1-inactivated melanocytomas (BIM), deep penetrating melanocytomas (DPM), atypical cellular blue nevi (ACBN), melanocytic tumors of uncertain malignant potential (MELTUMP), and pigmented epithelioid melanocytomas (PEM) were recategorized as high-grade intermediate. Subsequently, all lesions were reclassified according to the nine WHO pathways primarily based on provided genomic data. When distinctive genomic drivers were unavailable, lesions were reclassified based on the evaluation of available histopathology, ancillary tests, and additional case information. 


\section{Statistical analysis}

First, we created box plots to describe the data. Although these appeared not normally distributed, we also reported means to allow comparison with previously reported research on CNV counts. Second, we performed Mann-Whitney U tests to determine differences in $\mathrm{CNV}$ number between lesion categories and within classifications according to WHO pathway. Third, we created receiver operating characteristic (ROC) curves and calculated the $C$-statistic or area under the ROC curve (AUC). Fourth, sensitivity and specificity were calculated for a range of CNV cut-offs (0-7). As sensitivity analyses, we performed these analyses for two alternative categorizations of the lesions: (1) initially reported category and (2) considering low-grade intermediate lesions (BIN, CBN, CN with proliferative nodules, and DPN) as benign. Furthermore, we evaluated an alternative $\mathrm{CNV}$ count, including chromothripsis and $\mathrm{CN}-\mathrm{LOH}$. Also, we evaluated $\mathrm{CNV}$ count based on CGH data or SNP data only. Last, we evaluated sensitivity and specificity irrespective of $\mathrm{CNV}$ count by interpreting microarray data as positive for malignancy in the presence of CNVs suspect for melanoma, such as homozygous loss of 9p21 (CDKN2A) and gain of 11q13 (CCND1), 8q24 (MYC), or 6p25 (RREB1). All statistical analyses were performed in SPSS version 26.

\section{Results}

\section{Study selection}

Figure 1 shows the PRISMA flowchart for study selection [14]. The search yielded 647 hits, of which 432 were unique records. After assessment for eligibility, 25 studies were included in the meta-analysis, and a further six were identified through snowballing.

\section{Study characteristics}

The characteristics of the 31 included studies are listed in Table 1. All studies were either retrospective $(n=25)$, prospective $(n=4)$, or mixed $(n=2)$ case series. Twenty-two studies used CGH array, six studies used SNP array, and three studies used NGS. In total, data for 431 individual lesions were extracted, of which 252 (58.5\%) had been analyzed with CGH array, 144 (33.4\%) with SNP array, and 35 (8.1\%) with NGS.

\section{Recategorization and reclassification of lesions}

Initially, 113 lesions (26.2\%) were presented as benign, 212 (49.2\%) as ambiguous, and $106(24.6 \%)$ as malignant. Clinical follow-up was available for 297 lesions (68.9\%), of which 140 were ambiguous. Two benign and ten ambiguous lesions were recategorized as malignant based on follow-up with distant metastasis or additional case information. Per WHO-definition, 80 lesions were recategorized as low-grade intermediate (28 BIN, $22 \mathrm{CBN}, 15 \mathrm{CNPN}$, and $15 \mathrm{DPN}$ ) and 83 lesions as high-grade intermediate (30 ACBN, 7 BIM, 13 DPM, 16 MELTUMP, and 17 PEM). A total of 81 intermediate lesions could not be specified as either low- or highgrade (76 AST, three melanocytomas with CRTC1-TRIM11 fusions, and two melanocytomas with NRAS p.Q61R and IDH1 p.R132C mutations). After recategorization, 69 (16.0\%) benign, $244(56.6 \%)$ intermediate, and 118 (27.4\%) malignant lesions were available for meta-analysis. Distinctive genomic drivers, including $A L K, R O S 1, N T R K$, $B R A F$, or $M E T$ fusions and mutational status for $B A P 1$, $B R A F$, GNA11, GNAQ, HRAS, and NRAS, were available for 206/431 (47.8\%) lesions and 145/244 (59.4\%) intermediate lesions. Accordingly, 61/431 (14.1\%) lesions were reclassified, mostly "Spitz" lesions carrying a $B R A F$ p.V600E or NRAS p.Q61R mutation and lesions designated "DPN," "DPM," or "MELTUMP" carrying a GNAQ p.Q209L or GNA11 p.Q209L mutation.

\section{Chromosomal aberrations in intermediate lesions}

In our dataset, $18 / 69$ (26.1\%) of benign, 134/244 (54.9\%) of intermediate, and 112/118 (94.9\%) of malignant lesions displayed $\geq 1 \mathrm{CNV}$. Within intermediate lesions, 43/80 (53.8\%) of low-grade, 35/83 (42.2\%) of high-grade, and 56/ $81(69.5 \%)$ of intermediate lesions not otherwise specified (NOS) displayed $\geq 1 \mathrm{CNV}$. The most frequently encountered CNVs in intermediate lesions are listed in Table 2. Loss of 3p spanning the $B A P 1$ gene on $3 \mathrm{p} 21$ was most commonly found, all but one $(\mathrm{ACBN})$ harbored by BAPl-inactivated lesions. The most common gain involved 7q, carried mainly by AST. Chromosomal aberrations known to occur in melanoma $[7,9]$ frequently were infrequent or absent in intermediate lesions (marked with an asterisk in Table 2). Two AST displayed heterozygous loss of $9 \mathrm{p} 21$ spanning the CDKN2A gene. One ACBN showed a gain of 8q24 spanning the $M Y C$ gene. Aneuploidies were mainly found in BIN/BIM carrying a loss of chromosome 3 and CNPN carrying a loss of chromosome 7 and gain of chromosome 8. Chromothripsis was found in one malignant Spitz tumor (MST) and five intermediate lesions (two AST, one CBN, one CNPN, and one MELTUMP). Of these, clinical follow-up was only available for the CBN and CNPN. The CBN harbored chromothripsis of chromosomes 3 and 7 and 14 additional CNVs, without evidence of disease during a follow-up of 3.8 years. The CNPN harbored chromothripsis of $1 \mathrm{p}$ and two additional $\mathrm{CNVs}$, and the patient was disease-free at 3.5 years after excision. CN-LOH was found in eight melanomas and three intermediate lesions (one DPM, one MELTUMP, and one PEM). The DPM carried CN-LOH of 17q12-qter and did not harbor additional CNVs. The MELTUMP carried CN- 

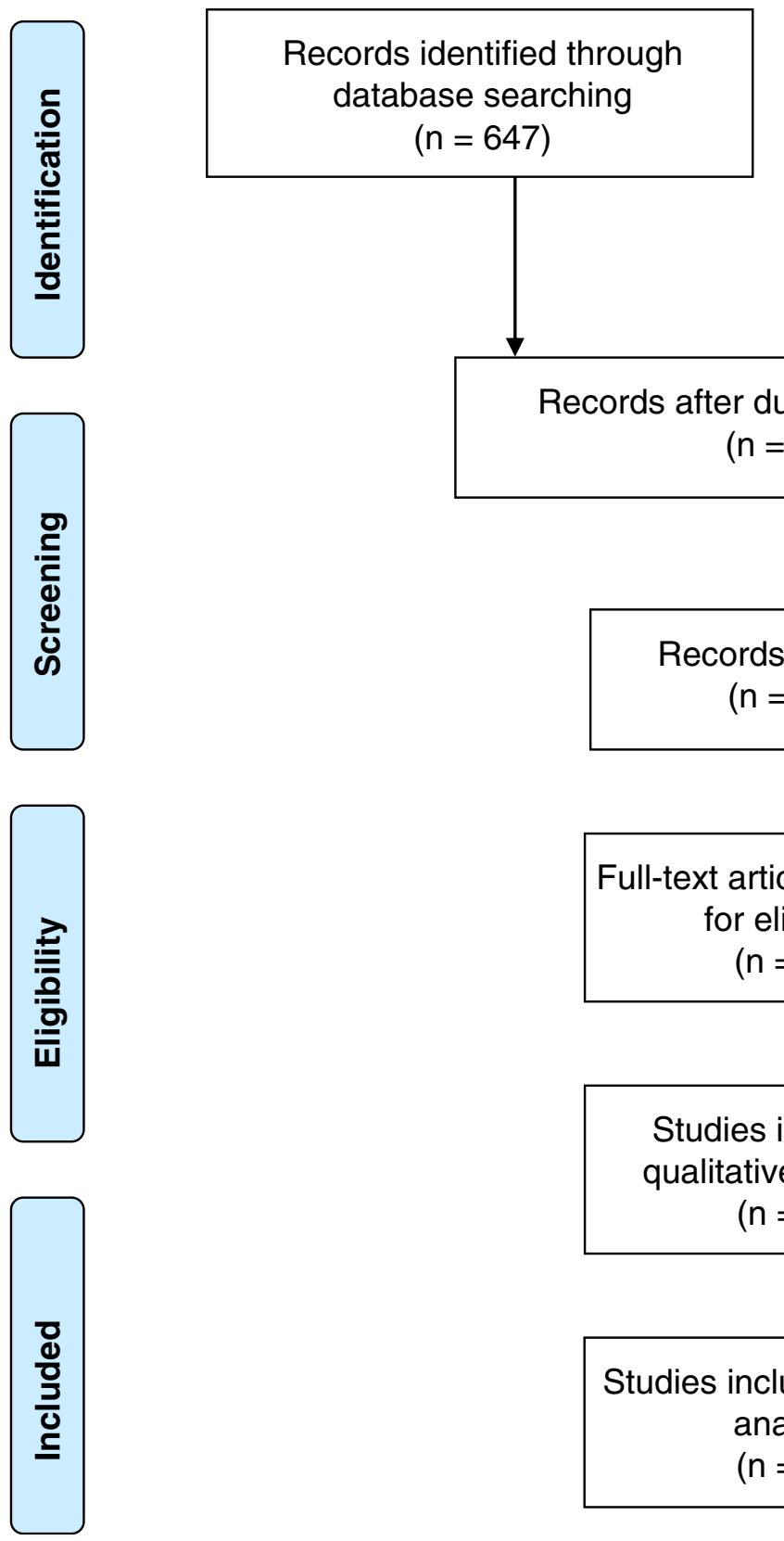

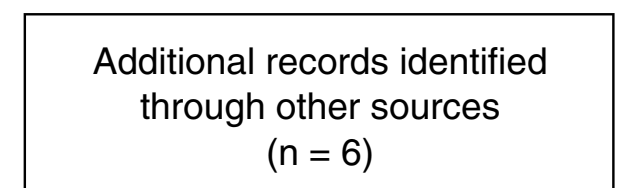

$$
=6)
$$
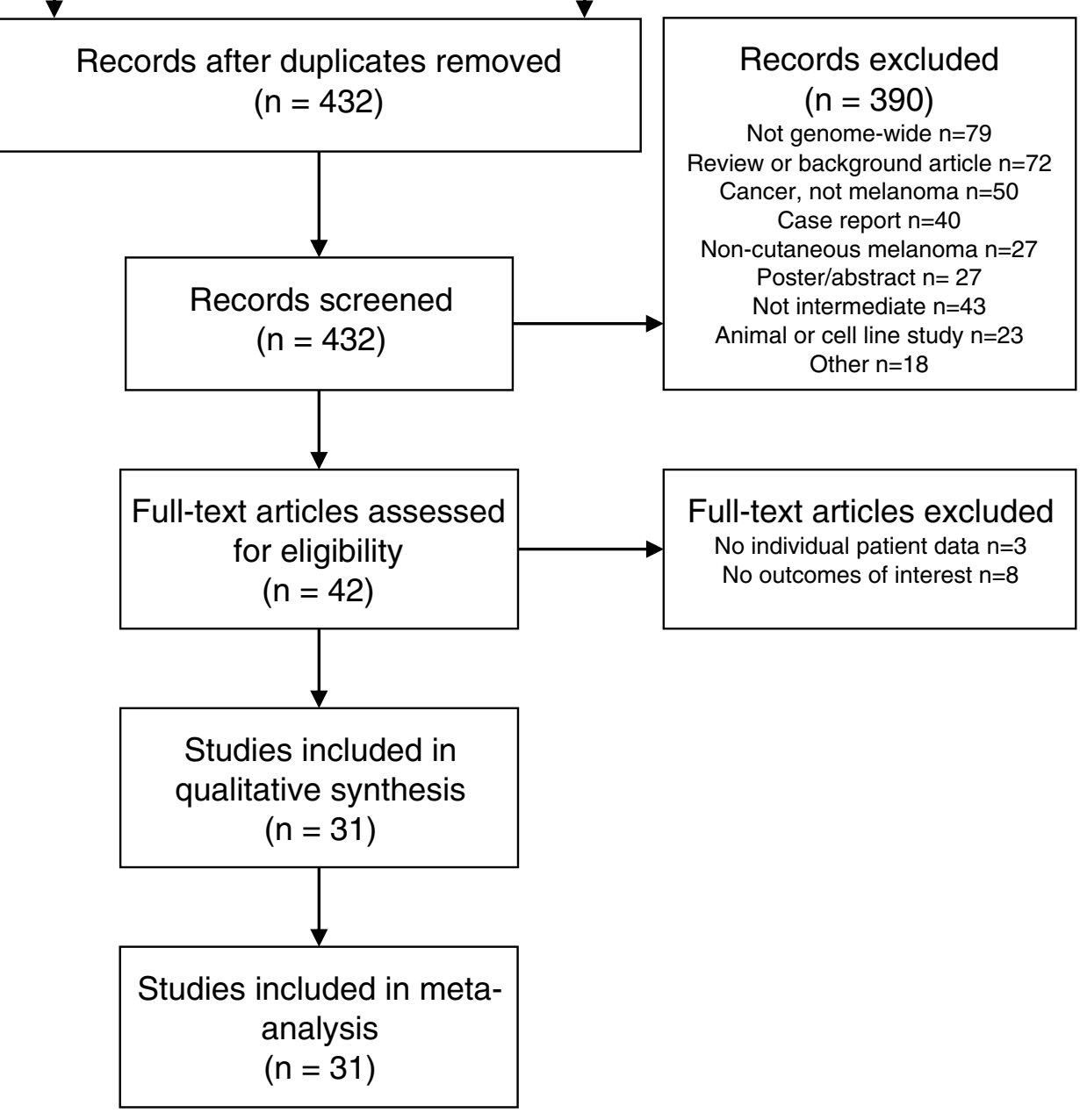

Full-text articles excluded

No individual patient data $n=3$

No outcomes of interest $n=8$

Fig. 1 PRISMA flow chart for study selection

LOH of chromosome 7 and had 15 additional CNVs. Clinical follow-up for these cases was not available. The PEM carried $\mathrm{CN}-\mathrm{LOH}$ of the distal part of chromosome $17 \mathrm{q}$ and did not harbor any additional CNVs. Short-term clinical follow-up (not specified) did not show any sign of disease.

\section{CNV counts after recategorization and reclassification}

Figures 2 and 3 show the number of CNVs per lesion category and WHO class, respectively. The CNV number in intermediate lesions (median 1, interquartile range [IQR] 0-2) was significantly higher $(p<0.001)$ compared to that in benign lesions (median 0, IQR 0-1) and significantly lower $(p<0.001)$ compared to that in malignant lesions (median 6, IQR 4-11) (Fig. 2). There was no significant difference between lowgrade or high-grade intermediate lesions $(p=0.499)$. In WHO pathway I, CNV number in BIM (median 1, IQR 1-1.5) was not significantly higher $(p=0.092)$ compared to that in $\mathrm{BIN}$ (median 1, IQR 1-1) and not significantly higher $(p=0.449)$ in DPM (median 0, IQR 0-1) compared to that in DPN (median 0, IQR 0-0). CNV number in PEM (median 0, IQR 0-0) was significantly lower $(p<0.001)$ compared to melanomas in PEM (median 4, IQR 4-5). In pathway IV, CNV number in AST (median 1, IQR 0-2) was significantly higher $(p<0.001)$ compared to that in Spitz nevi (median 0, IQR 0-1) and significantly lower $(p=0.001)$ compared to that in MSTs (median 
Table 1 Characteristics of included studies

\begin{tabular}{|c|c|c|c|c|c|c|}
\hline Study ID & Design & Method & N Ambiguous & N Benign & N Malignant & $\mathrm{N}$ Included \\
\hline Ali 2010 [15] & Retrospective case series & CGH array & 1 & 8 & 1 & 10 \\
\hline Alomari 2020 [16] & Mixed case series & SNP array & 34 & 21 & 40 & 95 \\
\hline Bastian 2002 [7] & Retrospective case series & CGH array & 9 & 13 & 6 & 29 \\
\hline Botton 2013 [17] & Retrospective case series & CGH array & 4 & 1 & 4 & 9 \\
\hline Busam 2014 [18] & Retrospective case series & CGH array & 2 & 0 & 0 & 2 \\
\hline Carter 2019 [19] & Retrospective case series & SNP array & 2 & 0 & 1 & 3 \\
\hline Cellier 2017. [20] & Retrospective case series & CGH array & 3 & 0 & 0 & 3 \\
\hline Chan 2016 [21] & Retrospective case series & CGH array & 12 & 5 & 9 & 26 \\
\hline Cohen 2017 [22] & Retrospective case series & NGS & 13 & 0 & 0 & 13 \\
\hline Cohen 2020 [23] & Retrospective case series & NGS & 2 & 0 & 5 & 7 \\
\hline Costa 2016 [24] & Retrospective case series & CGH array & 0 & 3 & 11 & 14 \\
\hline Fischer 2017 [25] & Retrospective case series & CGH array & 4 & 0 & 0 & 4 \\
\hline Gerami 2020 [26] & Retrospective case series & NGS* & 7 & 10 & 0 & 17 \\
\hline Hedayat 2017 [27] & Mixed case series & SNP array & 5 & 5 & 1 & 11 \\
\hline Held 2013 [28] & Retrospective case series & CGH array & 8 & 15 & 0 & 23 \\
\hline Houlier 2019 [29] & Retrospective case series & $\mathrm{CGH}$ array & 3 & 1 & 1 & 5 \\
\hline Hung 2016 [30] & Prospective case series & CGH array & 2 & 0 & 1 & 3 \\
\hline Lezcano 2020 [31] & Retrospective case series & SNP array & 1 & 0 & 1 & 2 \\
\hline Macagno 2020 [32] & Retrospective case series & CGH array & 2 & 0 & 0 & 2 \\
\hline Magro 2014 [33] & Prospective case series & CGH array & 6 & 0 & 0 & 6 \\
\hline Maize 2005 [34] & Retrospective case series & CGH array & 11 & 11 & 7 & 29 \\
\hline Raskin 2011 [35] & Prospective case series & CGH array & 16 & 8 & 3 & 27 \\
\hline Redon 2017 [36] & Retrospective case series & CGH array & 0 & 5 & 5 & 10 \\
\hline Reimann 2018 [37] & Prospective case series & $\mathrm{CGH}$ array & 3 & 0 & 2 & 5 \\
\hline Wiesner 2012 [38] & Retrospective case series & $\mathrm{CGH}$ array & 4 & 0 & 0 & 4 \\
\hline Yeh 2014 [39] & Retrospective case series & CGH array & 17 & 0 & 0 & 17 \\
\hline Yeh 2015 [40] & Prospective case series & $\mathrm{CGH}$ array & 16 & 0 & 3 & 19 \\
\hline Yeh 2016 [41] & Retrospective case series & $\mathrm{CGH}$ array & 6 & 2 & 0 & 8 \\
\hline Yeh 2019 [42] & Retrospective case series & $\mathrm{CGH}$ array & 11 & 5 & 3 & 19 \\
\hline Yeh-Botton 2015 [43] & Retrospective case series & $\mathrm{CGH}$ array & 4 & 0 & 2 & 6 \\
\hline Yelamos 2015 [44] & Retrospective case series & CGH array & 4 & 0 & 0 & 4 \\
\hline TOTAL & & & 212 & 113 & 106 & 431 \\
\hline
\end{tabular}

Study characteristics and number of included ambiguous, benign, and malignant lesions per study. *SNP array was used in two cases. $C G H$ comparative genomic hybridization, $S N P$ single nucleotide polymorphism, $N G S$ next-generation sequencing

5, IQR 4-8). In pathway VII, CNV number in CNPN (median 2 , IQR $1-5)$ was significantly higher $(p<0.001)$ compared to that in CN (median 0, IQR 0-0) and significantly lower $(p=0.009)$ compared to melanomas in $\mathrm{CN}$ (median 7.5, IQR 5.5-9.5). Last, in pathway VIII, CNV number in CBN (median 0 , IQR $0-0)$ was not significantly different $(p=0.585)$ from blue nevi (median 0, IQR $0-0$ ) but significantly lower $(p=0.015)$ compared to that in ACBN (median 0, IQR 0-2). CNV number in ACBN was significantly lower $(p<0.001)$ compared to melanomas in blue nevi (median 6, IQR 4-8) (Fig. 3). Relevant outliers in the intermediate category are shown as yellow dots in Fig. 2. The most extreme outlier corresponded to a CNPN harboring $22 \mathrm{CNVs}$, all gains and losses of whole chromosomes. Clinical follow-up was not available for the MELTUMP with $15 \mathrm{CNVs}$, the DPM with $10 \mathrm{CNVs}$, and the AST with $7 \mathrm{CNVs}$. The remaining lesions did not show evidence of disease during the available relatively short follow-up (varying from 14 to 46 months).

\section{CNV cut-off value}

The $C$-statistic to differentiate between nevi and melanoma was $0.96(95 \%$ CI $0.93-0.99, p<0.001)$ and between intermediate lesions and melanoma 0.90 (95\% CI $0.86-0.94$, $p<0.001$ ), indicating excellent ability to differentiate using $\mathrm{CNV}$ number [45]. In contrast, the CNV number displayed poor ability to differentiate between intermediate and benign lesions ( $C$-statistic $0.67,95 \%$ CI $0.61-0.73, p<0.001)$. 
Table 2 Most frequently found chromosomal aberrations in intermediate melanocytic lesions

\begin{tabular}{|c|c|c|c|c|c|c|c|c|c|}
\hline Losses & $\begin{array}{l}\text { Total } \\
(n=244)\end{array}$ & $\begin{array}{l}\text { Low-grade } \\
(n=83)\end{array}$ & $\begin{array}{l}\text { High-grade } \\
(n=83)\end{array}$ & $\begin{array}{l}\text { Intermediate } \\
\text { NOS }(n=81)\end{array}$ & Gains & $\begin{array}{l}\text { Total } \\
(n=244)\end{array}$ & $\begin{array}{l}\text { Low-grade } \\
(n=83)\end{array}$ & $\begin{array}{l}\text { High-grade } \\
(n=83)\end{array}$ & $\begin{array}{l}\text { Intermediate } \\
\text { NOS }(n=81)\end{array}$ \\
\hline $3 p$ & $10.2 \%$ & $20.0 \%$ & $9.6 \%$ & $1.2 \%$ & $7 q^{*}$ & $4.1 \%$ & $2.5 \%$ & $1.2 \%$ & $8.6 \%$ \\
\hline $1 p$ & $5.3 \%$ & $0.0 \%$ & $6.0 \%$ & $9.9 \%$ & $2 p$ & $2.9 \%$ & $1.3 \%$ & $0.0 \%$ & $7.4 \%$ \\
\hline Entire 3 & $5.3 \%$ & $13.8 \%$ & $2.4 \%$ & $0.0 \%$ & $1 \mathrm{p}$ & $2.0 \%$ & $0.0 \%$ & $2.4 \%$ & $3.7 \%$ \\
\hline $2 p$ & $3.3 \%$ & $1.3 \%$ & $2.4 \%$ & $6.2 \%$ & Entire 8 & $2.0 \%$ & $5.0 \%$ & $1.2 \%$ & $0.0 \%$ \\
\hline $6 q^{*}$ & $3.3 \%$ & $2.5 \%$ & $3.6 \%$ & $3.7 \%$ & $15 q$ & $2.0 \%$ & $0.0 \%$ & $6.0 \%$ & $0.0 \%$ \\
\hline Entire 9 & $3.3 \%$ & $2.5 \%$ & $4.8 \%$ & $2.5 \%$ & Entire 20 & $2.0 \%$ & $3.8 \%$ & $1.2 \%$ & $1.2 \%$ \\
\hline $9 p^{*}$ & $2.5 \%$ & $0.0 \%$ & $1.2 \%$ & $6.2 \%$ & Entire 7 & $1.6 \%$ & $1.3 \%$ & $1.2 \%$ & $2.5 \%$ \\
\hline $7 \mathrm{q}$ & $2.5 \%$ & $1.3 \%$ & $3.6 \%$ & $2.5 \%$ & $20 \mathrm{p}$ & $1.6 \%$ & $0.0 \%$ & $4.8 \%$ & $0.0 \%$ \\
\hline $15 q$ & $2.5 \%$ & $0.0 \%$ & $1.2 \%$ & $6.2 \%$ & $1 q^{*}$ & $1.2 \%$ & $0.0 \%$ & $2.4 \%$ & $1.2 \%$ \\
\hline $3 q$ & $2.0 \%$ & $0.0 \%$ & $4.8 \%$ & $1.2 \%$ & $6 \mathrm{p}^{*}$ & $1.2 \%$ & $1.3 \%$ & $2.4 \%$ & $0.0 \%$ \\
\hline $2 q$ & $1.6 \%$ & $1.3 \%$ & $0.0 \%$ & $3.7 \%$ & $9 q$ & $1.2 \%$ & $0.0 \%$ & $3.6 \%$ & $0.0 \%$ \\
\hline $6 p$ & $1.6 \%$ & $1.3 \%$ & $0.0 \%$ & $3.7 \%$ & $10 \mathrm{p}$ & $1.2 \%$ & $0.0 \%$ & $0.0 \%$ & $3.7 \%$ \\
\hline Entire 7 & $1.6 \%$ & $5.0 \%$ & $0.0 \%$ & $0.0 \%$ & Entire 15 & $1.2 \%$ & $2.5 \%$ & $0.0 \%$ & $1.2 \%$ \\
\hline
\end{tabular}

Most frequent gains and losses found in intermediate melanocytic lesions. Chromosomal aberrations are divided into gain or loss of entire chromosomes and segmental chromosomal aberrations (including focal aberrations and gain or loss of chromosomal arms). Chromosomal aberrations marked with an asterisk represent gains and losses frequently found in melanoma

Figure 4 shows sensitivity and specificity for differentiating intermediate from malignant lesions given various CNV cutoff values. Using a cut-off of $\geq 3 \mathrm{CNVs}, 85 \%$ of malignant lesions would be correctly categorized as malignant (sensitivity), and $84 \%$ of non-malignant lesions would be correctly classified as non-malignant (specificity).

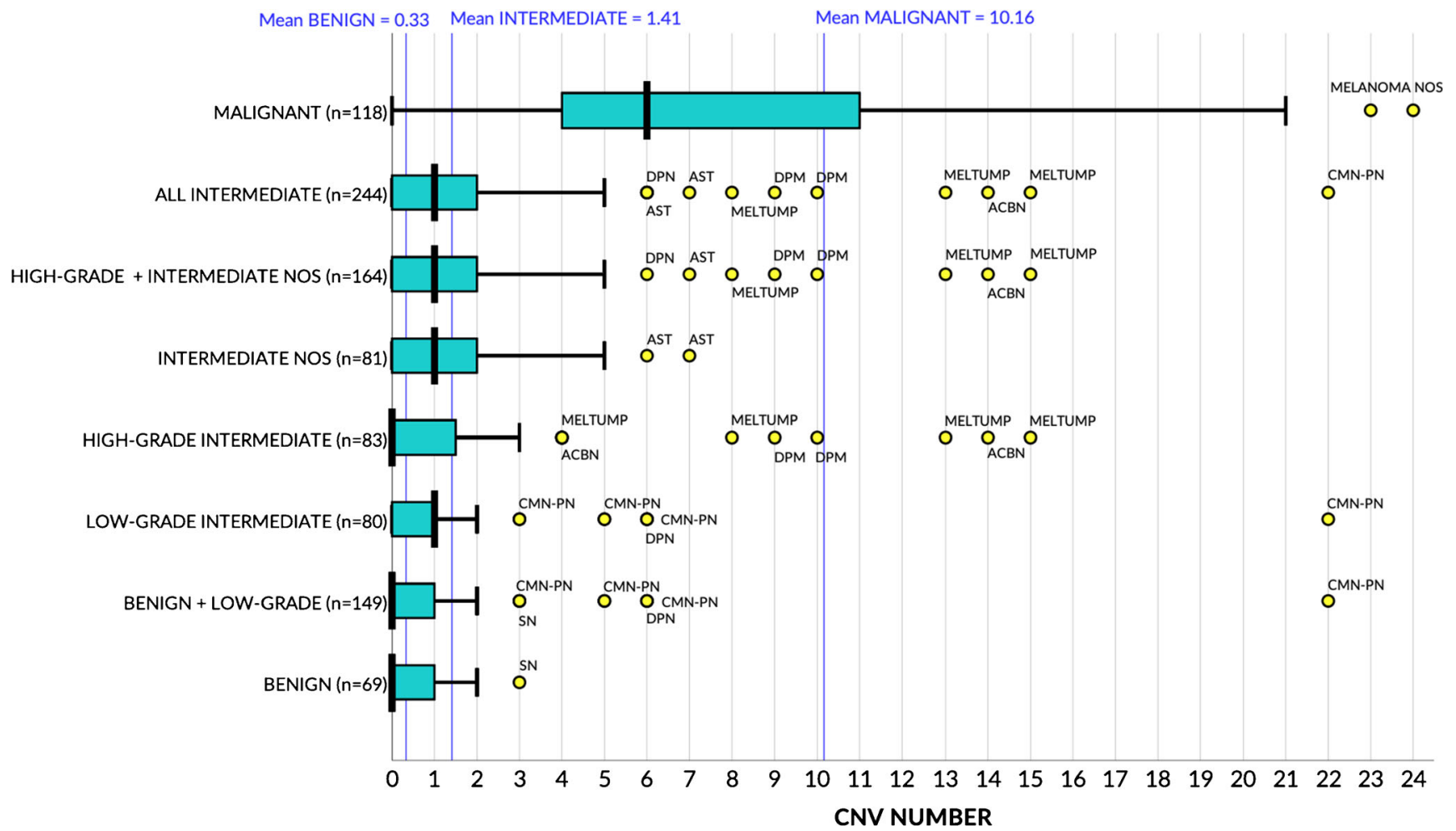

Fig. 2 Box and whisker plots showing CNV count after recategorization. Bold vertical black lines show the medians. The blue boxes show the interquartile range (first to third quartile), and the whiskers indicate the maximal value within 1.5 times the interquartile range. The vertical blue lines show the means of all benign, intermediate, and malignant lesions, and the yellow dots represent outliers. NB. Outliers in the malignant category with CNV counts $>24$ are not shown for clarity of the graph. ACBN, atypical cellular blue nevus; AST, atypical Spitz tumor; CMN$\mathrm{PN}$, congenital melanocytic nevus with proliferative nodule; DPN, deep penetrating nevus; DPM, deep penetrating melanocytoma; MELTUMP, melanocytic tumor of uncertain malignant potential; SN, Spitz nevus; Melanoma NOS, not otherwise specified 


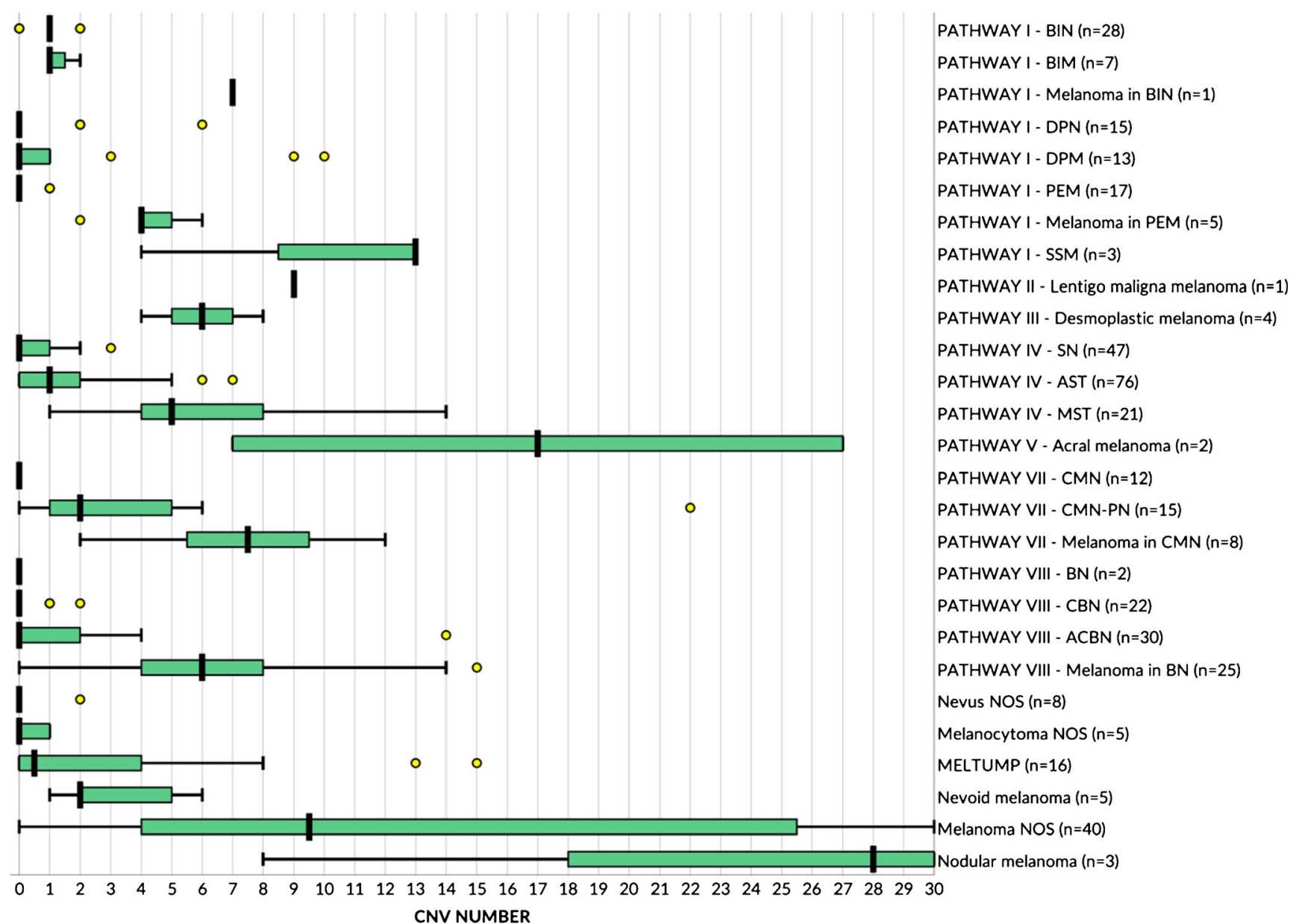

Fig. 3 Box and whisker plots showing CNV count after WHO reclassification. Bold vertical black lines show the medians. The green boxes show the interquartile range (first to third quartile), and the whiskers indicate the maximal value within 1.5 times the interquartile range. The yellow dots represent outliers. NB. CNV counts $>30$ are not shown for clarity of the graph. ACBN, atypical cellular blue nevus; AST, atypical Spitz tumor; BIN, BAP1-inactivated nevus; BIM, BAP1-

\section{Sensitivity analyses}

None of the alternative lesion categorizations or alternative $\mathrm{CNV}$ counts substantially changed the results for differentiation between intermediate and malignant lesions. The AUC based on the initially reported category was $0.88(95 \% \mathrm{CI}$ $0.84-0.92, p<0.001)$. The AUC when considering low-grade intermediate lesions benign remained 0.90 (95\% CI 0.860.94, $p<0.001)$. The AUC, when including chromothripsis and $\mathrm{CN}-\mathrm{LOH}$ in the CNV count, also remained 0.90 (95\% CI $0.86-0.94, p<0.001)$. The AUC based on SNP array or CGH data only decreased to 0.85 (95\% CI $0.78-0.92$, $p<0.001)$ and increased to 0.94 (95\% CI $0.91-0.97$, $p<0.001$ ), respectively. Including specific CNVs suspect for melanoma as a positive test marker did not substantially change these results. inactivated melanocytoma; $\mathrm{BN}$, blue nevus; $\mathrm{CBN}$, cellular blue nevus; CMN, congenital melanocytic nevus; CMN-PN, congenital melanocytic nevus with proliferative nodule; DPN, deep penetrating nevus; DPM, deep penetrating melanocytoma; MELTUMP, melanocytic tumor of uncertain malignant potential; PEM, pigmented epithelioid melanocytoma; MST, malignant Spitz tumor; NOS, not otherwise specified; SN, Spitz nevus; SSM, superficial spreading melanoma

\section{Risk of bias across studies}

The risk of bias was generally considered low to unknown and constituted mainly selection and information bias. Most studies used archival cases without adequately defining the selection process, creating an unknown selection bias risk. Three studies reported CNVs for selected representative cases. Comprising only 10 cases, we consider the impact of potential selection bias very low. In addition, the detection of CNVs is highly dependent on the type of microarray, resolution, DNA quality, and sample purity. Most studies used archival DNA from formalin-fixed paraffin-embedded (FFPE) tissue and did not report tumor cell percentages, which introduces an unknown risk of information bias. 


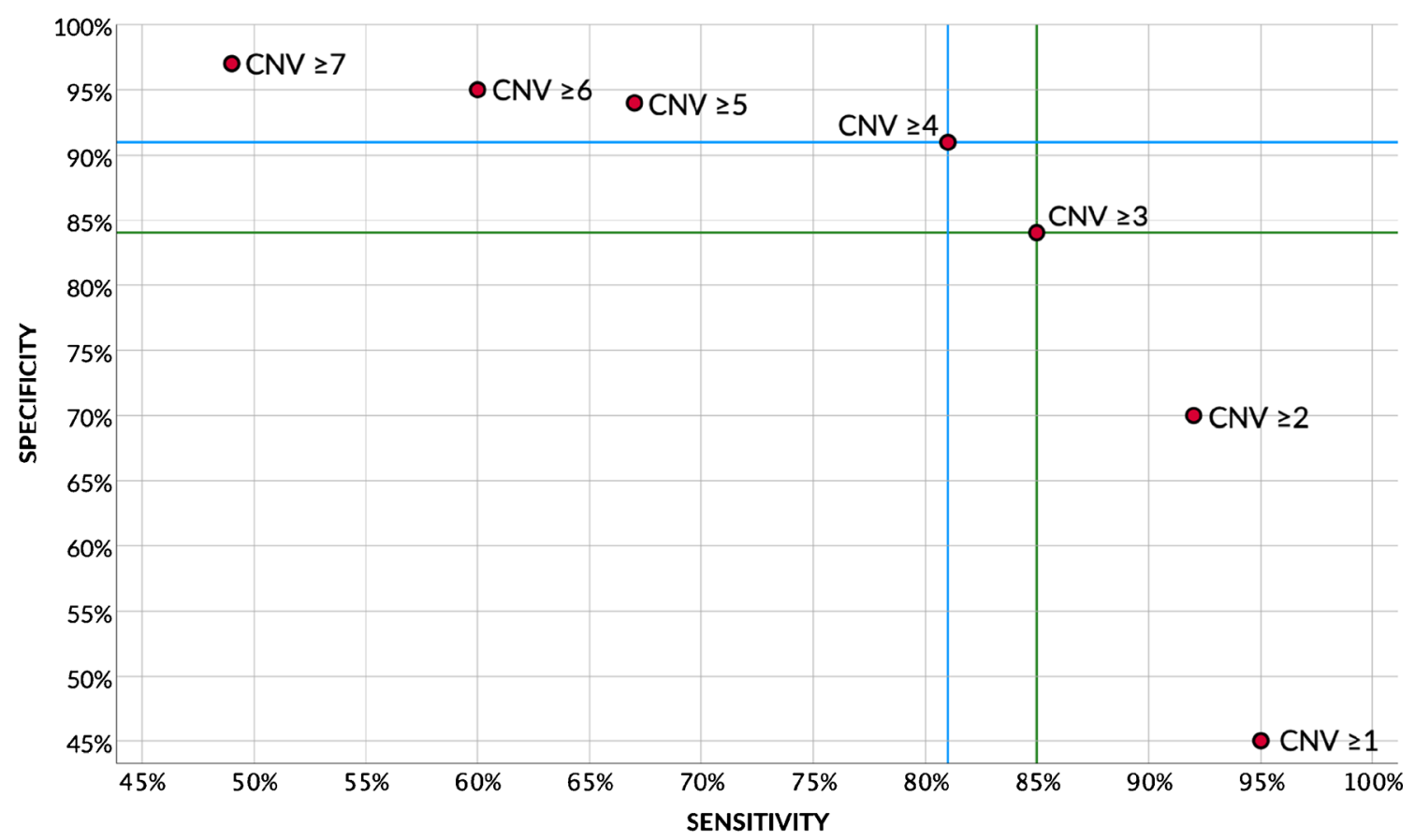

Fig. 4 Plot showing the trade-off between sensitivity (x-axis) and specificity (y-axis) to differentiate between intermediate and malignant lesions using different $\mathrm{CNV}$ cut-offs. Blue and green lines indicate sensitivity and

\section{Discussion}

To the best of our knowledge, this is the first systematic review and individual patient data meta-analysis to assess genome-wide CNVs as a diagnostic tool for intermediate melanocytic lesions, using cytogenetic tests such as SNP array and CGH array.

Chromosomal aberrations were found in $55.1 \%$ of intermediate lesions. Gains and losses frequently seen in melanoma, such as gain of $1 \mathrm{q}, 6 \mathrm{p}$, and $7 \mathrm{q}$ and loss of $6 \mathrm{q}$ and $9 \mathrm{p}$, were uncommon in intermediate lesions. $\mathrm{CN}-\mathrm{LOH}$ and chromothripsis were only found in intermediate and malignant lesions. Our analysis shows that the median number of CNVs in intermediate lesions is statistically significantly higher compared to that in nevi and lower compared to that in melanoma. Similarly, the number of CNVs significantly increased in WHO pathway IV (Spitz), VII (congenital), and VIII (blue) along the spectrum from nevus to melanoma. In contrast, the CNV number was not statistically different between BIN and BIM and between DPN and DPM. Surprisingly, "high-grade" melanocytomas (ACBN, BIM, DPM, PEM, and MELTUMP) carried CNVs less frequently than "low-grade" melanocytomas (BIN, CBN, CMN with proliferative nodules, and DPN). This observation demonstrates the difficulty of grading intermediate lesions using a four-tier system as is used in the WHO classification. Yet, our results suggest CNVs demonstrate excellent ability to differentiate between intermediate melanocytic lesions and melanoma in clinical practice. A specificity corresponding to CNV cut-offs of $\geq 4$ and $\geq 3$, respectively. $\mathrm{CNV}$, copy number variation

cut-off of $\geq 3 \mathrm{CNVs}$ corresponded to $85 \%$ sensitivity and $84 \%$ specificity, and a cut-off of $\geq 4 \mathrm{CNVs}$ corresponded to $81 \%$ sensitivity and $91 \%$ specificity, respectively.

Several CNV cut-offs for malignancy have previously been suggested. Based on their case series, Maize et al. and Chan et al. suggested a cut-off of $\geq 3 \mathrm{CNVs}$ and $\geq 4 \mathrm{CNVs}$, respectively, and Alomari et al. proposed an algorithm using $\geq 4$ significant CNVs with additional caveats in case of $\leq 3$ CNVs [16, 21, 34]. Our current meta-analysis integrates and expands their data, providing more robust evidence for various cut-offs in the classification of intermediate lesions. Both a cut-off of $\geq 3$ and $\geq 4 \mathrm{CNVs}$ can be considered, the first having a higher sensitivity (fewer false-negative diagnoses) and the latter having a higher specificity (fewer falsepositive diagnoses). Yet, sensitivity might prevail in clinical practice given the potentially disastrous consequences of a false-negative misdiagnosis, even at the cost of a modestly lower specificity and resulting treatment burden. Therefore, we propose a cut-off of $\geq 3 \mathrm{CNVs}$ as indicative of malignancy.

Of note, a minority of melanomas did not harbor CNVs, and benign lesions might carry $\mathrm{CNVs}$ with limited prognostic value. In contrast, specific CNVs may also be relevant if present in isolation, such as homozygous loss of 9p21 (CDKN2A) [16]. Therefore, CNVs should always be interpreted considering the clinicopathological context. Yet, the contextual interpretation of specific CNVs is difficult in unclassified lesions. For example, loss of 3p21 (BAPl) is insignificant in BIN/BIM but is of major significance in an ACBN or MEBN. It is 
currently mostly unknown which CNVs are most predictive for malignancy in the various WHO pathways, and this requires additional research.

The main strengths of this study include the following. All lesions have been vigorously reviewed based on published and unpublished individual patient data using the 2018 WHO classification of skin tumors. As such, our metaanalysis integrates the most recent clinicopathological and genomic insights to establish CNVs in intermediate lesions. Our analyses provide a better-defined CNV cut-off value for malignancy to support clinical decision-making, based on the largest pooled dataset of intermediate lesions to date. The sensitivity analyses strengthened the robustness of the results.

This study has several limitations. First, genome-wide microarray data are difficult to pool since the detection of CNVs is highly dependent on resolution and technical specifications. Second, the detection of CNVs depends on sample quality. Most studies used DNA from archival FFPE blocks and did not report the tumor cell percentages, although the latter should exceed $30 \%$ to detect CNVs reliably. Therefore, paucicellular lesions such as PEM and large-cell lesions such as MAP3K8-SVIL fused ASTs with strong lymphocytic infiltrate render dilution effects and make CNV detection more difficult. In our dataset, a minority of melanomas (5.1\%) did not carry any CNVs, and it is unclear if this is due to dilution or truly represents a lack of CNVs. This might have negatively influenced sensitivity, although our ROC analysis still showed excellent discriminative ability. Third, rules for CNV counts are not uniformly defined, and we were only able to count CNVs that were reported or provided by authors. CNVs reported to be attributable to a chromosomal fusion were counted as one CNV. This may have slightly overestimated the $\mathrm{CNV}$ number, especially in Spitz tumors, where fusions are a common driver event and probably not relevant for prognosis. Also, a minority of melanomas were reported to harbor extremely high CNV counts ( $>30)$, which likely included aberrations not included in our CNV count, such as chromothripsis. However, it is unlikely this substantially affected our results since we performed non-parametric tests. Fourth, clinical outcomes were not available in $34.0 \%$ of ambiguous cases. Follow-up with distant metastasis remains the gold standard for proof of malignancy and might still occur years after diagnosis. Yet, clinical follow-up was available for most outliers in the intermediate category. Fifth, distinctive driver mutations were available in $59.4 \%$ of intermediate lesions. For the remaining lesions, classification was based on histopathology alone, which is more subjective than genomic data. Last, we established our ROC analysis on one variable (CNV number), whereas ideally, diagnostic evaluation is performed via multivariable analysis, including all available diagnostic information. Nonetheless, it indicated excellent discriminative ability, which supports further research in a dedicated dataset. Despite these limitations, we believe this meta- analysis provides robust results applicable to general dermatopathology practice.

To conclude, this systematic review and individual patient data meta-analysis provides a comprehensive overview of $\mathrm{CNVs}$ in cutaneous intermediate melanocytic lesions and a diagnostic interpretation of different $\mathrm{CNV}$ cut-offs for malignancy, based on the largest pooled cohort of ambiguous melanocytic neoplasms to date. Our results suggest that a cut-off of $\geq 3$ CNVs might represent the optimal trade-off between sensitivity and specificity in clinical practice to distinguish intermediate from malignant lesions. Future research should externally validate this cut-off in a distinct dataset, assess the predictive value of specific CNVs in the various WHO pathways, and correlate genome-wide microarray data with objective genomic and clinical parameters.

Supplementary Information The online version contains supplementary material available at https://doi.org/10.1007/s00428-021-03095-5.

Author contribution Conceptualization: Chiel Ebbelaar, Anne Jansen, and Willeke Blokx. Methodology: Chiel Ebbelaar, Lourens Bloem, and Willeke Blokx. Formal analysis: Chiel Ebbelaar and Lourens Bloem. Data curation: Chiel Ebbelaar Anne Jansen, and Willeke Blokx. Writing — original draft preparation: Chiel Ebbelaar. Writing-review and editing: Anne Jansen, Lourens Bloem, and Willeke Blokx. Visualization: Chiel Ebbelaar. All authors have read and agreed to the published version of the manuscript.

\section{Declarations}

Conflict of interest The authors declare no competing interests.

\section{References}

1. Bastian BC (2014) The molecular pathology of melanoma: an integrated taxonomy of melanocytic neoplasia. Annu Rev Pathol 9: 239-271

2. Shain AH, Yeh I, Kovalyshyn I, Sriharan A, Talevich E, Gagnon A, Dummer R, North J, Pincus L, Ruben B, Rickaby W, D'Arrigo C, Robson A, Bastian BC (2015) The genetic evolution of melanoma from precursor lesions. N Engl J Med. 373(20):1926-1936

3. Shain AH, Joseph NM, Yu R et al (2018) Genomic and transcriptomic analysis reveals incremental disruption of key signaling pathways during melanoma evolution. Cancer Cell 34(1): 45-55.e4

4. Elder DE, Massi D, Scolyer RA, Willemze R (2018) WHO classification of skin tumours, 4th edn. International Agency for Research on Cancer (IARC), Lyon Cedex 08 (France), Lyon

5. Elmore JG, Barnhill RL, Elder DE et al (2017) Pathologists' diagnosis of invasive melanoma and melanocytic proliferations: observer accuracy and reproducibility study. BMJ j2813:357

6. Gerami P, Busam K, Cochran A, Cook MG, Duncan LM, Elder DE, Fullen DR, Guitart J, LeBoit PE, Mihm MC, Prieto VG, Rabkin MS, Scolyer RA, Xu X, Yun SJ, Obregon R, Yazdan P, Cooper C, Weitner BB, Rademaker A, Barnhill RL (2014) Histomorphologic assessment and interobserver diagnostic 
reproducibility of atypical spitzoid melanocytic neoplasms with long-term follow-up. Am J Surg Pathol 38:934-940

7. Bastian BC, Olshen AB, LeBoit PE, Pinkel D (2003) Classifying melanocytic tumors based on DNA copy number changes. Am J Pathol 163:1765-1770

8. Gerami P, Scolyer RA, Xu X, Elder DE, Abraham RM, Fullen D, Prieto VG, LeBoit PE, Barnhill RL, Cooper C, Yazdan P, Guitart J, Liu P, Pestova E, Busam K (2013) Risk assessment for atypical spitzoid melanocytic neoplasms using FISH to identify chromosomal copy number aberrations. Am J Surg Pathol. 37(5):676-684

9. Mesbah Ardakani N, Thomas C, Robinson C, Mina K, Harvey NT, Amanuel B, Wood BA (2017) Detection of copy number variations in melanocytic lesions utilising array based comparative genomic hybridisation. Pathology 49:285-291

10. Ouzzani M, Hammady, Zbys Fedorowicz Z, Elmagarmid A (2016) Rayyan, a web and mobile app for systematic reviews. Systematic Reviews 5:210

11. Beaubier N, Tell R, Huether R et al (2018) Clinical validation of the Tempus xO assay. Oncotarget 9(40):25826-25832

12. Talevich E, Shain AH, Botton T et al (2016) CNVkit: Genomewide copy number detection and visualization from targeted DNA sequencing. PLoS Comput Biol 12(4):e1004873

13. Lallas A, Kyrgidis A, Ferrara G, Kittler H, Apalla Z, Castagnetti F, Longo C, Moscarella E, Piana S, Zalaudek I, Argenziano G (2014) Atypical Spitz tumours and sentinel lymph node biopsy: a systematic review. Lancet Oncol. 15(4):e178-e183

14. Moher D, Liberati A, Tetzlaff J, Altman DG, PRISMA Group (2009) Preferred reporting items for systematic reviews and metaanalyses: the PRISMA statement. PLoS Med 6(7):e1000097

15. Ali L, Helm T, Cheney R, Conroy J, Sait S, Guitart J, Gerami P (2010) Correlating array comparative genomic hybridization findings with histology and outcome in spitzoid melanocytic neoplasms. Int J Clin Exp Pathol. 3(6):593-599

16. Alomari AK, Miedema JR, Carter MD, Harms PW, Lowe L, Durham AB, Fullen DR, Patel RM, Hristov AC, Chan MP, Wang M, Andea AA (2020) DNA copy number changes correlate with clinical behavior in melanocytic neoplasms: proposal of an algorithmic approach. Mod Pathol. 33(7):1307-1317

17. Botton T, Yeh I, Nelson T, Vemula SS, Sparatta A, Garrido MC, Allegra M, Rocchi S, Bahadoran P, McCalmont TH, LeBoit PE, Burton EA, Bollag G, Ballotti R, Bastian BC (2013 Nov) Recurrent BRAF kinase fusions in melanocytic tumors offer an opportunity for targeted therapy. Pigment Cell Melanoma Res. 26(6):845-851

18. Busam KJ, Kutzner H, Cerroni L, Wiesner T (2014) Clinical and pathologic findings of Spitz nevi and atypical Spitz tumors with ALK fusions. Am J Surg Pathol. 38(7):925-933

19. Carter MD, Durham AB, Miedema JR, Harms PW, Chan MP, Patel RM, Lowe L, Fullen DR, Hristov AC, Wang M, Andea AA (2019) Molecular testing of borderline cutaneous melanocytic lesions: SNP array is more sensitive and specific than FISH. Hum Pathol. $86: 115-123$

20. Cellier L, Perron E, Pissaloux D, Karanian M, Haddad V, Alberti L, de la Fouchardière A (2018) Cutaneous melanocytoma with CRTC1-TRIM11 fusion: report of 5 cases resembling clear cell sarcoma. Am J Surg Pathol. 42(3):382-391

21. Chan MP, Andea AA, Harms PW, Durham AB, Patel RM, Wang M, Robichaud P, Fisher GJ, Johnson TM, Fullen DR (2016) Genomic copy number analysis of a spectrum of blue nevi identifies recurrent aberrations of entire chromosomal arms in melanoma ex blue nevus. Mod Pathol. 29(3):227-239

22. Cohen JN, Joseph NM, North JP, Onodera C, Zembowicz A, LeBoit PE (2017) Genomic analysis of pigmented epithelioid melanocytomas reveals recurrent alterations in PRKAR1A, and PRKCA genes. Am J Surg Pathol. 41(10):1333-1346

23. Cohen JN, Yeh I, Mully TW, LeBoit PE, McCalmont TH (2020) Genomic and clinicopathologic characteristics of PRKAR1A- inactivated melanomas: toward genetic distinctions of animal-type melanoma/pigment synthesizing melanoma. Am J Surg Pathol. 44(6):805-816

24. Costa S, Byrne M, Pissaloux D, Haddad V, Paindavoine S, Thomas L, Aubin F, Lesimple T, Grange F, Bonniaud B, Mortier L, Mateus C, Dreno B, Balme B, Vergier B, de la Fouchardiere A (2016) Melanomas associated with blue nevi or mimicking cellular blue nevi: clinical, pathologic, and molecular study of 11 cases displaying a high frequency of GNA11 mutations, BAP1 expression loss, and a predilection for the scalp. Am J Surg Pathol. 40(3): 368-377

25. Fischer AS, High WA (2018) The difficulty in interpreting gene expression profiling in BAP-negative melanocytic tumors. J Cutan Pathol. 45:659-666. https://doi.org/10.1111/cup.13277. Epubaheadofprint

26. Gerami P, Kim D, Compres EV et al (2020) Clinical, morphologic, and genomic findings in ROS1 fusion Spitz neoplasms. Mod Pathol. https://doi.org/10.1038/s41379-020-00658-w

27. Hedayat AA, Linos K, Jung HS, Tafe LJ, Yan S, LeBlanc RE, Lefferts JA (2017) Evaluating melanocytic lesions with single nucleotide polymorphism (SNP) chromosomal microarray. Exp Mol Pathol. 103(3):279-287

28. Held L, Eigentler TK, Metzler G, Leiter U, Messina JL, Glass LF, Garbe C, Bauer J (2013) Proliferative activity, chromosomal aberrations, and tumor-specific mutations in the differential diagnosis between blue nevi and melanoma. Am J Pathol. 182(3):640-645

29. Houlier A, Pissaloux D, Masse I, Tirode F, Karanian M, Pincus LB, McCalmont TH, LeBoit PE, Bastian BC, Yeh I, de la Fouchardière A (2020) Melanocytic tumors with MAP3K8 fusions: report of 33 cases with morphological-genetic correlations. Mod Pathol. 33(5): $846-857$

30. Hung T, Argenyi Z, Erickson L, Guitart J, Horenstein MG, Lowe L, Messina J, Piepkorn MW, Prieto VG, Rabkin MS, Schmidt B, Selim MA, Shea CR, Trotter MJ, Barnhill RL (2016) Cellular blue nevomelanocytic lesions: analysis of clinical, histological, and outcome data in 37 cases. Am J Dermatopathol. 38(7):499-503

31. Lezcano C, Jungbluth AA, Busam KJ (2020) Comparison of immunohistochemistry for PRAME with cytogenetic test results in the evaluation of challenging melanocytic tumors. Am J Surg Pathol. 44(7):893-900

32. Macagno N, Pissaloux D, Etchevers H, Haddad V, Vergier B, Sierra-Fortuny S, Tirode F, de la Fouchardière A (2020) Cutaneous melanocytic tumors with concomitant NRASQ61R and IDH1R132C mutations: a report of 6 cases. Am J Surg Pathol. 44(10):1398-1405

33. Magro CM, Abraham RM, Guo R, Li S, Wang X, Proper S, Crowson AN, Mihm M (2014) Deep penetrating nevus-like borderline tumors: a unique subset of ambiguous melanocytic tumors with malignant potential and normal cytogenetics. Eur J Dermatol. 24(5):594-602

34. Maize JC Jr, McCalmont TH, Carlson JA et al (2005) Genomic analysis of blue nevi and related dermal melanocytic proliferations. Am J Surg Pathol. 29(9):1214-1220

35. Raskin L, Ludgate M, Iyer RK, Ackley TE, Bradford CR, Johnson TM, Fullen DR (2011) Copy number variations and clinical outcome in atypical Spitz tumors. Am J Surg Pathol. 35(2):243-252

36. Redon S, Guibourg B, Talagas M, Marcorelles P, Uguen A (2018) A diagnostic algorithm combining immunohistochemistry and molecular cytogenetics to diagnose challenging melanocytic tumors. Appl Immunohistochem Mol Morphol. 26(10):714-720

37. Reimann JDR, Salim S, Velazquez EF, Wang L, Williams KM, Flejter WL, Brooke L, Sunder S, Busam KJ (2018) Comparison of melanoma gene expression score with histopathology, fluorescence in situ hybridization, and SNP array for the classification of melanocytic neoplasms. Mod Pathol. 31(11):1733-1743 
38. Wiesner T, Murali R, Fried I, Cerroni L, Busam K, Kutzner H, Bastian BC (2012) A distinct subset of atypical Spitz tumors is characterized by BRAF mutation and loss of BAP1 expression. Am J Surg Pathol. 36(6):818-830

39. Yeh I, Mully TW, Wiesner T, Vemula SS, Mirza SA, Sparatta AJ, McCalmont TH, Bastian BC, LeBoit PE (2014) Ambiguous melanocytic tumors with loss of 3p21. Am J Surg Pathol. 38(8): 1088-1095

40. Yeh I, de la Fouchardiere A, Pissaloux D, Mully TW, Garrido MC, Vemula SS, Busam KJ, LeBoit PE, McCalmont TH, Bastian BC (2015) Clinical, histopathologic, and genomic features of Spitz tumors with ALK fusions. Am J Surg Pathol. 39(5):581-591

41. Yeh I, Tee MK, Botton T, Shain AH, Sparatta AJ, Gagnon A, Vemula SS, Garrido MC, Nakamaru K, Isoyama T, McCalmont TH, LeBoit PE, Bastian BC (2016) NTRK3 kinase fusions in Spitz tumours. J Pathol. 240(3):282-290

42. Yeh I, Busam KJ, McCalmont TH et al (2019) Filigree-like rete ridges, lobulated nests, rosette-like structures, and exaggerated maturation characterize Spitz tumors with NTRK1 fusion. Am J Surg Pathol. 43(6):737-746

43. Yeh I, Botton T, Talevich E, Shain AH, Sparatta AJ, de la Fouchardiere A, Mully TW, North JP, Garrido MC, Gagnon A, Vemula SS, McCalmont TH, LeBoit PE, Bastian BC (2015) Activating MET kinase rearrangements in melanoma and Spitz tumours. Nat Commun. 6:7174

44. Yélamos O, Arva NC, Obregon R, Yazdan P, Wagner A, Guitart J, Gerami P (2015) A comparative study of proliferative nodules and lethal melanomas in congenital nevi from children. Am J Surg Pathol. 39(3):405-415

45. Pencina MJ, D'Agostino RB Sr (2015) Evaluating discrimination of risk prediction models: The C Statistic. JAMA. 314(10):1063-1064

Publisher's note Springer Nature remains neutral with regard to jurisdictional claims in published maps and institutional affiliations. 